\title{
Standards for Validating Stress Analyses by Integrating Simulation and Experimentation
}

\author{
Erwin Hack ${ }^{1}$, George Lampeas ${ }^{2}$, John Mottershead ${ }^{3}$, Eann Patterson ${ }^{4}$, Thorsten Siebert ${ }^{5}$ and \\ Maurice Whelan ${ }^{6}$ \\ ${ }^{1}$ Laboratory of Electronics/Metrology/Reliability, EMPA, Duebendorf, Switzerland \\ ${ }^{2}$ Department of Mechanical Engineering and Aeronautics, University of Patras, Greece \\ ${ }^{3}$ Department of Mechanical Engineering, University of Liverpool, UK \\ ${ }^{4}$ Composite Vehicle Research Center, Michigan State University, East Lansing, MI 48824, \\ USA. eann@egr.msu.edu \\ ${ }^{5}$ Dantec Dynamics GmbH, Ulm, Germany \\ ${ }^{6}$ Institute for Health and Consumer Protection, European Commission DG Joint Research \\ Centre, Italy.
}

\begin{abstract}
A reference material and a series of standardized tests have already been developed for respectively calibrating and evaluating optical systems employed for measuring in-plane static strain (for draft standard see: www.twa26.org). New work has commenced on the design of a reference material (RM) for use with instruments or systems capable of measuring three-dimensional displacements and strains during dynamic events. The rational decision-making process is being utilized and the initial stages have been completed, i.e. the identification and weighting of attributes for the design, brain-storming candidate designs and evaluation of candidate designs against the attributes. Twenty-five attributes have been identified and seven selected as being essential in any successful design, namely: the boundary conditions must be reproducible; a range of in-plane and out-of-plane displacement values must be present inside the field of view; the RM must be robust and portable; there is a means of verifying the performance in situ; and for cyclic loading it must be possible to extract data throughout the cycle. More than thirty candidate designs were generated and have been reduced to nine viable designs for further evaluation. In parallel with this effort to design a reference material, work is also in progress to optimize methodologies for conducting analyses via both simulations and experiments. Image decomposition methods are being explored as a means to making quantitative comparisons full-field data maps from simulations and experiments in order to provide a comprehensive validation procedure.
\end{abstract}

\section{INTRODUCTION}

\subsection{Engineering context}

The Olympic motto is "Citius, Altius, Fortius" which translates as "Faster, Higher, Stronger"; for the modern design engineer this could be modified only slightly to "Faster, Lighter, Stronger". In an era where global competition dominates industry, everyone wants everything to be faster so that you can gain edge on your competitors and generally speaking it is easier to achieve swiftness with lighter designs while the dangers inherent in great speed imply the need for greater strength. Lighter and stronger also provide advantages in terms of environmental footprint since lighter usually implies more energy efficient both in terms of service and manufacturing resources, and stronger offers the potential for a longer life cycle. In the quest for "Faster, Lighter, Stronger" structural analysis plays a crucial part in optimizing the performance of devices, machines and vehicles of all types and ensuring that safety requirements are achieved. In most design processes, computational modeling is the dominant form of structural analysis and so the reliability of the model becomes a critical issue for engineers involved in making design decisions. Schwer [1] has described in outline a 'Guide for verification and validation in 
computational solid mechanics' [2]. In this context verification is defined as being two processes: first, identifying and eliminating errors of logic and programming from the code used for modeling; and second, quantifying the errors arising from the code as a consequence of discretizations required for the modeling. So verification can be largely performed without reference to the real-world whereas validation is concerned with establishing how well the model represents the real-world, at least in the context of the anticipated use of the model for solid mechanics or more particularly, design. It is recommended that validation should be achieved by reference to experiments conducted specifically for this purpose but the guide provides no insight or guidelines for the conduct of such experiments. This is not surprising since the computational mechanics community was responsible for the guide's preparation; however, the experimental mechanics community has not been idle in this regard and made a first step with a draft proposed standard for the calibration and evaluation of optical system for in-plane, static strain measurement in 2007 [3]. An outline description of this proposed standard is provided below and then work in progress to extend it to include three-dimensional measurements in dynamic loading cases is reported.

\subsection{Calibration and evaluation of measurement systems for in-plane, static strain}

Technical Working Area 26: Full-field Optical Stress and Strain Measurement [4] of VAMAS [5] was formed in 1999 with the aim of bringing together those concerned with the use of optical techniques for full field measurements of stress and strain in order to develop internationally accepted standards. In 2002 a consortium of European organisations embracing universities, research laboratories, instrument manufacturers, end users and national laboratories was formed to pursue SPOTS (Standardisation Project for Optical Techniques for Strain measurement) [6]. SPOTS was an EC shared cost RTD contract (no. G6RD-CT-2002-00856 (SPOTS)) which lasted for three years and in 2006 issued a proposed standard for calibration and assessment of optical strain measurement systems $[4,6]$. This document has been endorsed by VAMAS TWA26 following independent international review and is currently awaiting recommendation by VAMAS to ISO.

The SPOTS standard relates to optical systems designed for making measurements of static strain over a field of view which can be approximated to a plane. A reference material, shown in figure 1, and accompanying experimental protocol are provided for the calibration of such instruments at any scale. Indeed the standard recommends that the calibration should be conducted at the same scale as the planned experiment and for the same range of strain values.

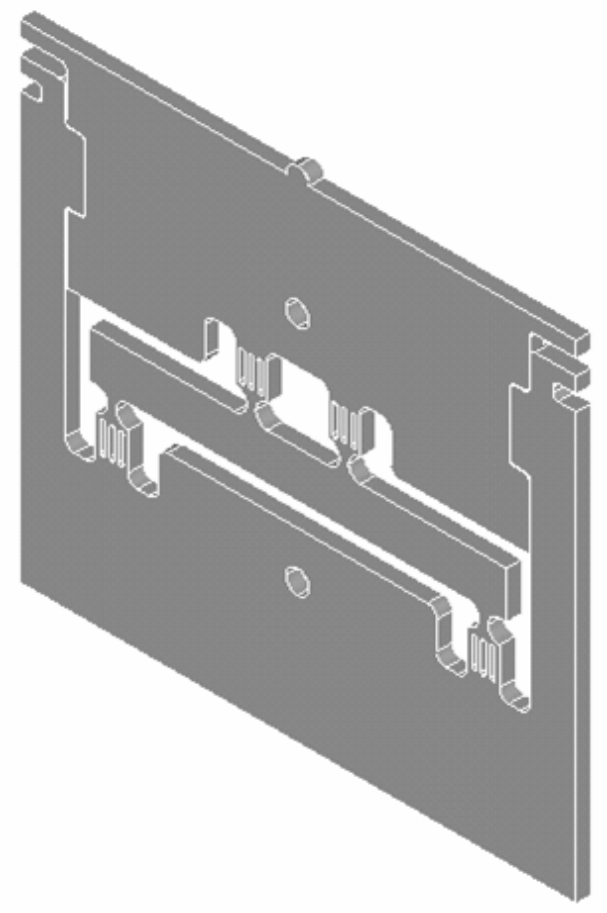

Figure 1: Three-dimensional view of the physical reference material (EU Community Design Registration 000213467) which is scalable to any size and can be manufactured in any material. (CSPOTS consortium) 
Briefly, the reference material consists of a monolithic frame surrounding a beam subject to four-point bending. The gauge section of the reference material is the central portion of the beam. The beam is connected to the frame by a series of whiffle-trees which are designed to minimize the constraints applied to the beam. The frame ensures that the boundary and loading conditions are easily reproducible which earlier round-robins had demonstrated were a limiting issue in making comparisons between datasets [7]. The load can be applied in compression by placing the reference material on a platen and loading the nipple on the top surface, or in tension using the two circular holes along its center-line. The load is monitored as a relative displacement of the upper and lower portions of the frame which can be measured via a calibrated displacement transducer at the top left and top right corners. The use of calibrated displacement transducers provides the first link in the chain of traceability to the length standard. The importance of traceability in this context has been discussed by Hack et al [8]. The flowchart in figure 2 illustrates the experimental protocol for the use of the reference material and an exemplar of its use is provided by Whelan et al [9]. The values to be reported from the calibration process are highlighted in a box on the right of the flow chart and include the uncertainties in the calibration which are the minimum uncertainties that would be obtained in any subsequent experiment using the calibrated instrument. These uncertainties allow confidence limits to be defined for measured strain data which significantly improves the quality of data provided for the validation process.

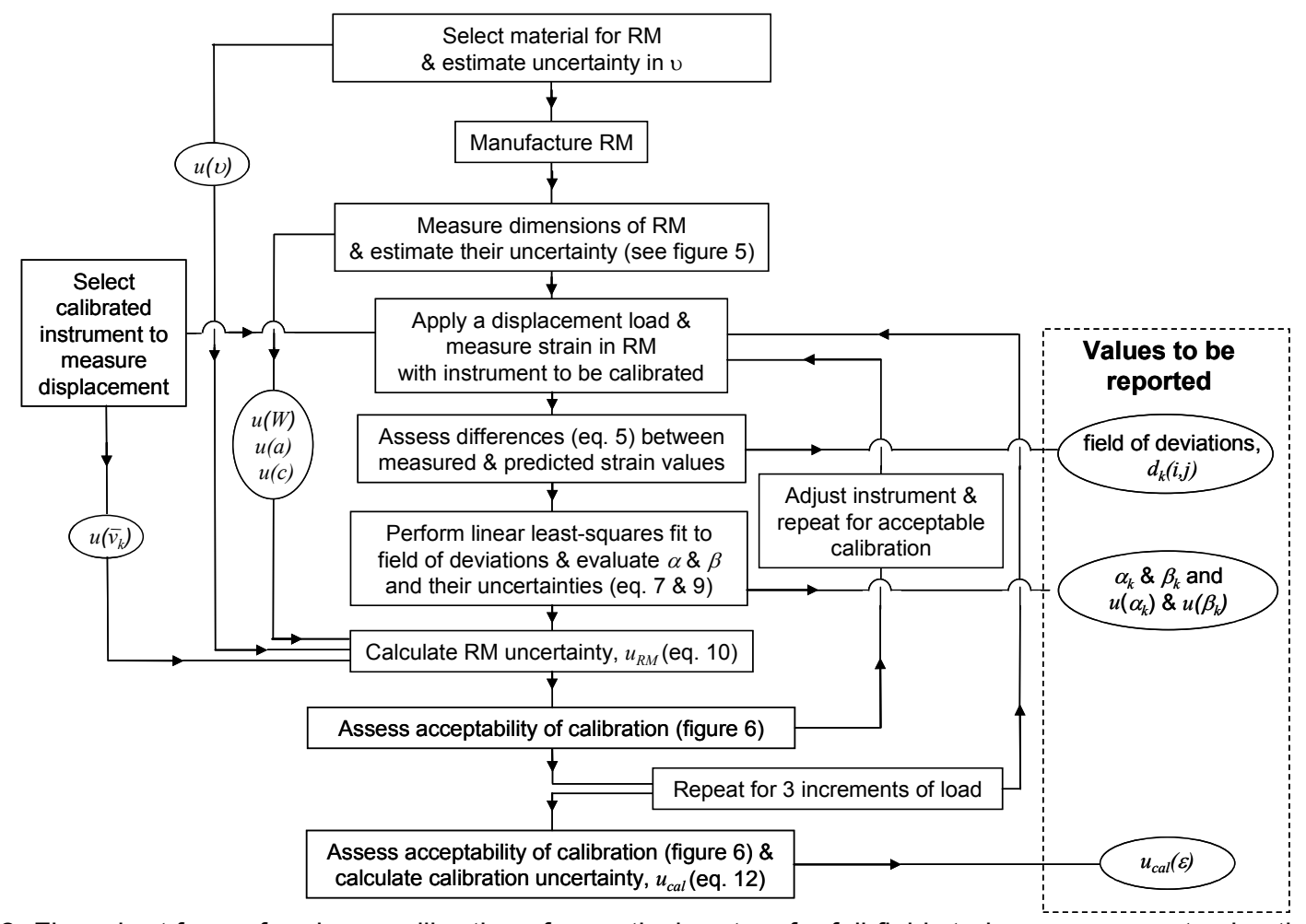

Figure 2: Flow-chart for performing a calibration of an optical system for full-field strain measurement using the SPOTS reference material where $u$ is uncertainty, $W, a$ and $c$ are characteristic dimensions, $v$ is Poisson ratio and $\alpha$ and $\beta$ are fit parameters relating to the correlation of the experimental and analytical strain distribution for the gauge section of the beam. References to figures and equations relate to the SPOTS standard found in [6] (๑ SPOTS consortium)

The SPOTS consortium also provided the design and protocol for a standardised test material $[6,10]$ for use in evaluating an optical system against its design specification or other instruments. The strain distribution in the gauge section of the standardised test material is significantly more complicated than the simple linear distribution in the reference material and is designed to be a challenge to the capabilities of the most sophisticated instrument. As shown in figure 3 , the gauge section consists of a disc in contact with a semi-infinite half-plane and is surrounded by a monolithic frame as in the reference material. The standardised test material can only be loaded in compression and contains the same feature for protecting the gauge section from overload and for monitoring the displacement load although in this case traceability to the length standard is not required. The rigid motion implicit in the contact loading presents difficulties for many strain measurement techniques and can be reduced substantially by loading the test material upside down as shown in figure 3 . However, it should be noted that many real-world scenarios will involve significant rigid body motion. 


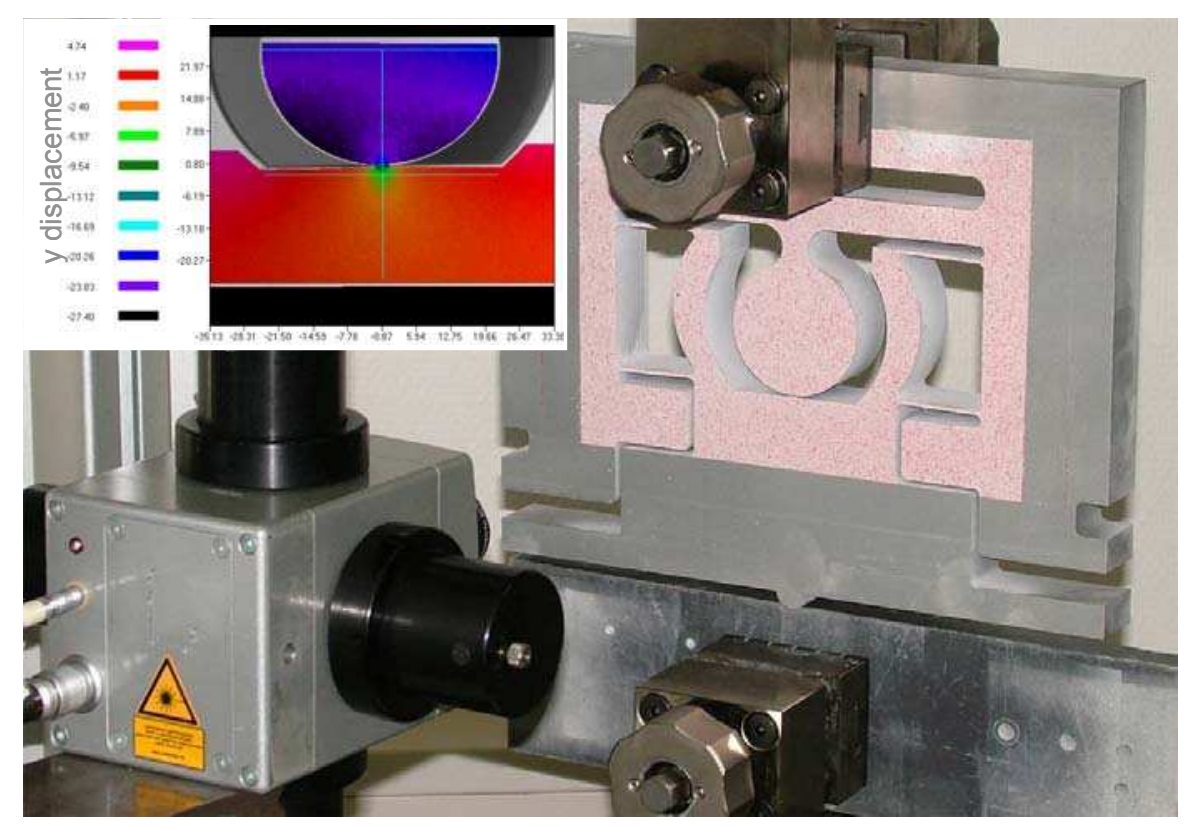

Figure 3: A physical standardised test material (EU Community Design Registration 000299094) manufactured from aluminium having a disc diameter of $50 \mathrm{~mm}$ being tested using an ESPI system (Dantec Dynamics Q300) with typical results shown inset. (@ SPOTS consortium)

\section{VALIDATION OF DYNAMIC ANALYSES}

\subsection{The ADVISE Project}

The SPOTS project was completed in early 2006 and a new consortium was formed during 2008 under the title of ADVISE (Advanced Dynamic Validations using Integrated Simulation and Experimentation) [11] with the purpose of extending the work into three dimensions and dynamic loading. ADVISE is a three-year program partly funded by the EU $7^{\text {th }}$ Framework Programme. The members were selected to provide continuity from the SPOTS project, to create an international collaboration and to span the innovation process from concept through product development and manufacture to end-use. The partners in ADVISE are: Airbus (UK), Centro Ricerche Fiat (Italy), Dantec Dynamics GmbH (Germany), EC Joint Research Centre, EMPA Swiss Federal Laboratories for Materials Testing and Research (Switzerland), High Performance Space Structure Systems GmbH (Germany), Michigan State University (USA), University of Liverpool (UK) and the University of Patras (Greece).

The goals of the ADVISE project are: to develop a reference material and associated protocol for the calibration of optical systems capable of measuring displacements in a wide range of dynamic applications so that a system can be certified and the measurement uncertainties quantified; and to develop a methodology for making quantitative comparisons between full-field data sets from such systems and computational models. Composite structures used in the transport industry are being used as a vehicle for testing these capabilities.

\subsection{Design of a reference material}

The rational decision making model [12] is being employed to guide the design of the new reference material following its successful use in the SPOTS project and because it has the considerable advantage of providing clear opportunities for input by the wider experimental mechanics community. In outline, this model involves the definition and subsequent weighting, in terms of importance, of attributes that the design either must (essential attributes) or should (desirable attributes) possess. Candidate designs are then developed, often through brainstorming, and then evaluated on the extent to which they possess the attributes. Designs that do not or could not be modified to possess all of the essential attributes are discarded and the remainder are ranked based on the weighted sum of the degree of possession of each attribute. The top ranked designs are taken forward for detailed embodiment and further evaluation. 


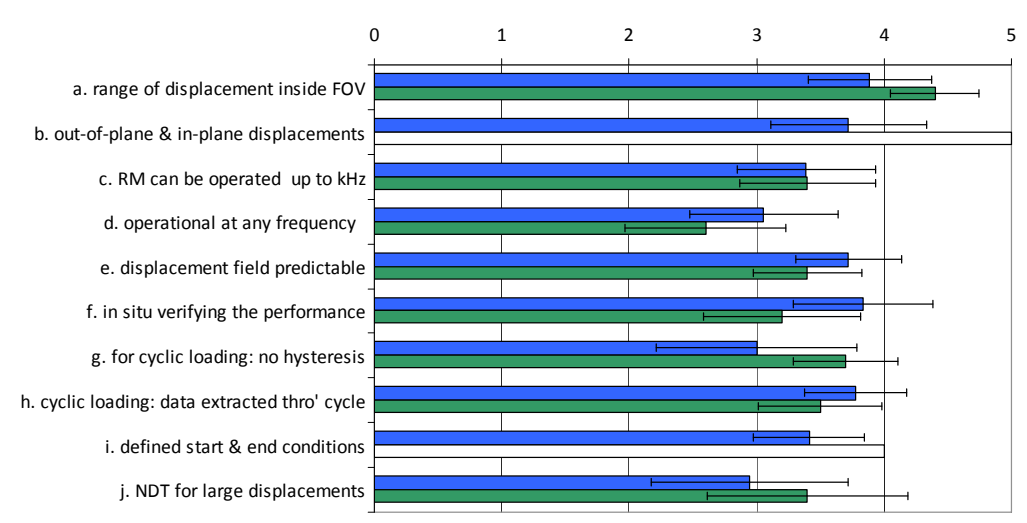

Figure 4: Attributes and their weightings for the displacement field in the dynamic reference material. The weightings ( 1 - unimportant, 2 - preferred, 3 - important, 4 - highly desirable, or 5 - essential) assigned by individual partners in the project were averaged (green) and the same exercise repeated for the wider community (blue). Some attributes were suggested by the community during the weighting process (white) and so were not weighted by a significant number of participants. (@ ADVISE consortium)

The ADVISE consortium developed a set of attributes in early 2009 which were presented to the community at a VAMAS TWA26 workshop held at the Society for Experimental Mechanics Conference in Albuquerque, NM in June 2009. Participants at the workshop and members of the Society were invited by email to suggest additional attributes and to weight them. The results are presented in figures 4 and 5 together with the weightings based on input from the ADVISE partners. The essential attributes were chosen as those for which the sum of the mean and a standard deviation was greater than 4.0 when the data from the ADVISE consortium was merged with that from the community. The essential attributes are listed below:

- $\quad$ there is a range of displacement values inside the field of view

- in-plane and out-of-plane displacements available

- there is a means of verifying the performance in situ

- for cyclic loading: data can be extracted throughout the cycle

- the boundary conditions are reproducible

- it is portable

- it is robust

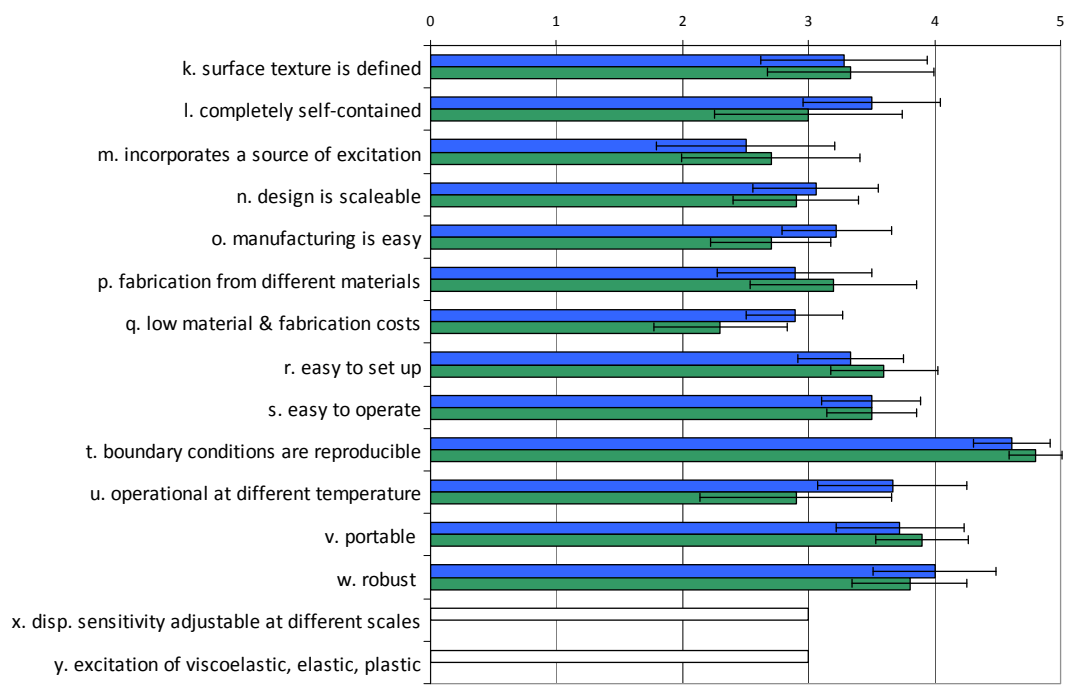

Figure 5: Attributes and their weightings for the physical embodiment of the dynamic reference material. The weightings (1 unimportant, 2 - preferred, 3 - important, 4 - highly desirable, or 5 - essential) assigned by individual partners in the project were averaged (green) and the same exercise repeated for the wider community (b/ue). Some attributes were suggested by the community during the weighting process (white) and so were not weighted by a significant number of participants.

(@) ADVISE consortium) 
The ADVISE consortium met in Fall 2009 to brainstorm candidate designs for the reference material that would be appropriate for cyclic loading, single high-speed loading events that result in deformations that vary linearly with load and single high-speed loading events that result in deformations that vary non-linearly with load. More than thirty candidate designs were generated but after assessment for the possession of the essential attributes only nine designs have been retained for further evaluation. At the time of writing these designs are undergoing evaluation involving both experimentation and computational modeling in order to gain a full understanding of their potential, prior to selecting a final design for detailed development and preparation of an accompanying protocol for its use.

\section{DISCUSSION AND CONCLUSIONS}

A guide for the verification and validation of computational solid mechanics was published in $2006[1,2]$ and has been complemented by a proposed standard for the calibration and evaluation of optical systems for full-field strain measurement $[3,4,6]$. The latter is applicable only to in-plane, static strain distributions but nevertheless represented a substantial effort that included one of the first attempts to quantify the uncertainties involved in measuring strain over a wide field of view.

A new project was launched in late 2008 to extend the proposed standard to include three-dimensional strain analysis in dynamic cases. A single reference material is being sought that would allow calibration of optical systems capable of measuring displacement and, or strain in three dimensional components subject to dynamic loading which might or might not be cyclical and that may or may not induce non-linear responses. This is wide and challenging design brief which is being tackled with the aid of the rational decision making model and with input from the community. The consortium undertaking the development has an international membership with representation from across the innovation process and appears to be broadly representative of the wider experimental mechanics community as indicated by the excellent correlation of the weighting of design attributes shown in figure 6.

The new design of reference material is due for completion in late 2011 and following a period of testing within the ADVISE consortium will be available via a round-robin for more comprehensive tests within the wider community. A protocol for its use and the reporting of results will also be produced. In parallel, research is underway to establish viable methods for making quantitative comparisons of large data fields generated from computational and experimental approaches to structural analysis. Ultimately it is expected that this new work will be incorporated into a revised draft standard by VAMAS TWA26.

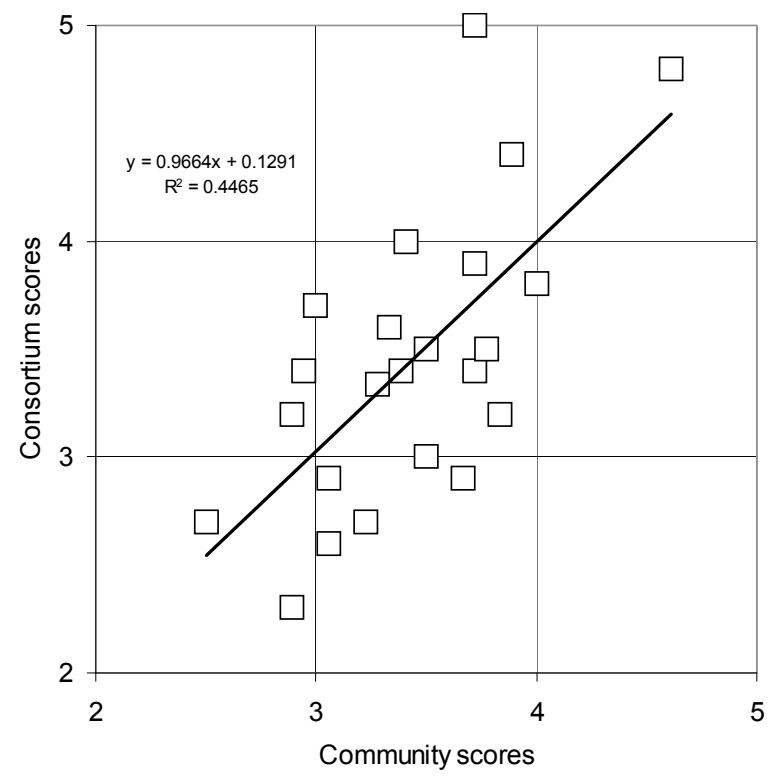

Figure 6: Correlation of the weightings by the ADVISE consortium partners and the members of the scientific community of the attributes required or desired in the dynamic reference material. (๑ ADVISE consortium) 


\section{ACKNOWLEDGEMENTS}

The authors cheerfully acknowledge the inputs of the following persons in the ADVISE project: Richard Burguete, Mara Feligiotti, Alexander Ihle, George Lampeas, John Mottershead, Andrea Pipino, Hans Reinhard Schubach, Thorsten Siebert, and Victor Wang. The ADVISE project is a Seventh Framework Programme Collaborative Project within Theme 7: Transportation including Aeronautics (Grant no. 218595) funding of which is gratefully acknowledged.

\section{REFERENCES}

1. L.E. Schwer, An overview of the PTC 60/V\&V 10: guide for verification and validation in computation solid mechanics', Engineering with Computers, 23:245-252 (2007)

2. ASME V\&V 10-2006, Guide for verification and validation in computational solid mechanics, American Society of Mechanical Engineers, New York, 2006.

3. E.A. Patterson, E. Hack, P. Brailly, R.L. Burguete, Q. Saleem, T. Siebert, R.A. Tomlinson, M.P. Whelan, 'Calibration and evaluation of optical systems for full-field strain measurement', Optics and Lasers in Engineering, 45(5):550-564, 2007.

4. www.twa26.org

5. www.vamas.org

6. www.opticalstrain.org

7. D. -A. Mendels, E. Hack, P. Siegmann, E.A. Patterson, L. Salbut, M. Kujawinska, H.R. Schubach, M. Dugand, L. Kehoe, C. Stochmil, P. Brailly, M.P. Whelan, 'Round robin exercise for optical strain measurement', Proc. $12^{\text {th }}$ Int. Conf. Exptl. Mechanics, Advances in Experimental Mechanics edited by C. Pappalettere, McGraw-Hill, Milano, pp.695-6, 2004.

8. E. Hack, R.L. Burguete, E.A. Patterson, 'Traceability of optical techniques for strain measurement', Proc. BSSM Int. Conf. on Advanced Experimental Mechanics, Southampton, UK, published as Applied Mechanics \& Materials, vols.3-4, pp.391-396, 2005.

9. M.P. Whelan, D. Albrecht, E. Hack, E.A. Patterson, 'Calibration of a speckle interferometry full-field strain measurement system', Strain, 44(2):180-190, 2008.

10. E.A. Patterson, P. Brailly, R.L. Burguete, E. Hack, T. Siebert, M.P.Whelan, 'A challenge for high performance full-field strain measurement systems', Strain, 43(3):167-180, 2007.

11. www.dynamicvalidation.org

12. N. Cross, Engineering Design Methods (John Wiley \& Sons, London, 1989) 